\title{
Loop-Mediated Isothermal Amplification Assay for Rapid and Reliable Detection of Mycobacterium tuberculosis in Sputum Samples
}

\author{
Sharma $\mathrm{BK}^{1^{*}}$, Pandey BD${ }^{2}$, Sharma $\mathrm{K}^{3}$, Sapkota $\mathrm{B}^{4}$, Singh $\mathrm{A}^{5}$, Adhikari $\mathrm{N}^{5}$, Maharjan $\mathrm{B}^{6}$ \\ ${ }^{1}$ Department of Microbiology, Gandaki Medical College and Teaching Hospital, Pokhara, Nepal \\ ${ }^{2}$ Sukraraj Tropical and Infectious Disease Hospital \& Everest International Clinic and Research Center, Kathmandu, Nepal \\ ${ }^{3}$ Department of Radiology, Gandaki Medical College \& Teaching Hospital, Pokhara, Nepal \\ ${ }^{4}$ Department of Research and Biotechnology, Anandaban Hospital, Kathmandu, Nepal \\ ${ }^{5}$ Department of Microbiology, Tribhuvan University, Kirtipur, Nepal \\ ${ }^{6}$ German- Nepal Tuberculosis Project (GENETUP), Kathmandu, Nepal
}

\section{Keywords}

Culture,

Loop-Mediated Isothermal Amplification, Microscopy, Mycobacterium tuberculosis, Polymerase Chain Reaction.

\section{Corresponding author}

*Dr. Binita Koirala Sharma, PhD

Lecturer, Department of Microbiology

Gandaki Medical College \& Teaching

Hospital, Pokhara, Nepal

Email: binita_keshab@yahoo.com

\begin{abstract}
Introduction: Tuberculosis (TB) remains a major global health problem. The most common method for diagnosing TB in developing countries is sputum smear microscopy; however, the sensitivity of this test is relatively lower. Detection of Mycobacterium tuberculosis using conventional culture and biochemical-based assays is time-consuming and laborious. Polymerase Chain Reaction (PCR) is also available for diagnosis of Mycobacterium tuberculosis. However, the PCR assay requires an expensive thermal cycler to amplify the DNA fragment in multiple temperature-dependent steps. Therefore, a simple and sensitive method for rapid detection has been anxiously awaited. The loop-mediated isothermal amplification (LAMP) assay is a diagnostic technique which can aid in the fight against TB in resource-poor countries. The LAMP assay can amplify a targeted sequence at a constant temperature. Therefore, a large and costly thermal cycler is not necessary for a LAMP assay.
\end{abstract}

Objectives: The objective of this study was to identify Mycobacterium tuberculosis directly from sputum by LAMP and to compare its efficacy over routinely used methods.

Methods: A total of 106 (53 fluorochrome staining positive and 53 fluorochrome staining negative) sputum samples were collected in this study. Mycobacterial DNA was extracted from concentrated sputum samples by freezing and boiling method. LAMP assay using a set of six specific primers targeting the M. tuberculosis $16 \mathrm{~S}$ rRNA gene with high sensitivity was used to analyze sputum samples. The results were then compared with that of the culture method, which was considered as the gold standard method.

Results: Among total of 106 samples studied by microscopy and culture, 53 were positive by both, whole four were positive by culture but negative by microscopy. With reference to culture, the microscopy had sensitivity $92.98 \%$, specificity $100 \%$, and predictive value of positive 
test $100 \%$, predictive value of negative test $92.5 \%$. Out of 106 samples subjected to culture and LAMP for the diagnosis of TB, 55 samples were positive by both tests and two were positive only in culture, while 48 were negative in both tests and one was negative only in culture. While comparing the LAMP with culture as a gold standard, the sensitivity of LAMP was $96.49 \%$, specificity was $97.95 \%$, predictive value of positive test was $98.21 \%$, predictive value of negative test was $96 \%$.

Conclusions: Comparative experiments showed that the LAMP assay is a rapid, sensitive, and specific method to detect M. tuberculosis infection. Indeed, an inexpensive LAMP assay would be potential as a diagnostic test for tuberculosis, especially in resource-limited settings.

\section{INTRODUCTION}

Tuberculosis (TB) remains a major global health problem. Although the worldwide incidence and prevalence of TB are gradually decreasing, approximately 1.5 million deaths a year are attributed to TB according to the World Bank and the World Health Organization ${ }^{1}$. The major challenge in combating TB is the lack of a rapid, reliable, and inexpensive diagnostic test for detection of Mycobacterium tuberculosis.

Early diagnosis is important for the control and prevention of TB. Currently, the most common method for TB diagnosis worldwide is sputum smear microscopy, the sensitivity of which is notoriously poor, particularly in human immunodeficiency virus (HIV) positive patients ${ }^{2,3}$. Culture methods can detect as few as 100 Mycobacterium cells per one $\mathrm{ml} \mathrm{specime \textrm {n } ^ { 4 }}$ and TB culture is still considered the gold standard for the detection of Mycobacterium tuberculosis. However, the culture methods require up to several weeks in sophisticated facilities, such as biosafety level 3 , and delays in the diagnosis of ТВ may harm many patients due to the current prevalence of multidrug resistant TB (MDR-TB) ${ }^{5}$. To address the need for rapid and sensitive diagnosis of $\mathrm{TB}$, a number of nucleic acid amplification assays have been invented $6,7,8$; however, they are still not routinely applied in developing countries due to their high cost, complicated procedures, insufficient laboratory facilities, and a shortage of skilled technologists $9,10,11$.

To overcome the limitations of current molecular techniques, a new molecular-biological technique, known as loop mediated isothermal amplification (LAMP), was developed by Notomi et $a l^{12}$. As the LAMP technique uses strand-displacing DNA polymerases that have high activity under isothermal temperatures ranging from 60 to $68^{\circ} \mathrm{C}$, no expensive equipment is required. The
LAMP reaction produces stem-loop DNA structures with several inverted repeats and cauliflower- like structures with multiple loops. The products of amplified LAMP reactions can be measured by a change in the turbidity of the reaction mixture because magnesium pyrophosphate accumulates in the reaction mixture as a byproduct of the amplification reaction ${ }^{13}$. The LAMP assay does not require the use of complicated procedures, equipment or machines. Therefore, the LAMP assay is considered more rapid, simpler and more economical than other TB diagnostic methods. An inexpensive LAMP has the potential to be adopted for the diagnosis of tuberculosis in peripheral laboratories and resource-limited settings. The aim of this study was to identify Mycobacterium tuberculosis directly from sputum by LAMP and to compare its efficacy over routinely used methods.

\section{METHODS}

\section{Study design and clinical sputum specimens}

This study was carried out from October 2006 to November 2007 at National TB Reference Lab GermanNepal Tuberculosis Project (GENETUP), Kalimati; Mycobacterial Research Laboratory, Anandaban Hospital; Everest International Clinic and Research Center (EICRC), Kathmandu in collaboration with Osaka Perfectural Institute of public health, Japan. A total of 106 sputum samples were collected and divided into two groups i.e. group A and group B. In group A, 53 sputum samples were collected from the new cases of pulmonary tuberculosis patients and Group B includes 53 acid fast bacelli negative sputum samples from patients who had complains of chest pain, cough and fever were collected at GENETUP. During sample and data collection, study objectives and expected outcomes were explained to 
each study participant before receiving their consent. About four ml of muco-purulent early morning sample was collected, followed on the same day by microscopy and sample culture preparation.

Smear microscopy was performed according to the acid-fast bacillus stains, with auramine-O staining. Grading of microscopy was done as suggested by WHO, $1998 \mathrm{a}^{14}$. The remaining sample after treated with $\mathrm{N}$-acetyl-L-cysteine - $\mathrm{NaOH}$ method to kill the undesirable microorganisms other than the AFB. Decontaminated sputum of $0.1 \mathrm{ml}$ was inoculated into each of the two culture tubes containing 2\% Ogawa medium and incubated at $37^{\circ} \mathrm{C}$. When colonies were observed during incubation, acid fastness of bacilli was determined by a smear test. When no colonies appeared after weekly observation for eight weeks, the result was considered negative. Grading of primary culture was done as suggested by WHO, 1998b ${ }^{15}$.

\section{Loop-Mediated Isothermal Amplification (LAMP)}

\section{Sample Treatment for LAMP:}

Methods performed by Sapkotal et al was adopted to treat the concentrated sputum samples for LAMP. Briefly, an equal volume of $2 \% \mathrm{NaOH}$ and $0.5 \% \mathrm{~N}$-acetyl-L-cysteine (NaLC) was added to the concentrated sputum sample. These were mixed by inverting for several times and left for 10 minutes to thinning out the sputum. The sample was then centrifuged at 12,000 rpm for 15 minutes. The supernatant was discarded and the pellet was resuspended in $50 \mathrm{mM}$ Tris $\mathrm{HCl}$ of $\mathrm{pH} 8.3$ equivalent to not less than $1 / 20^{\text {th }}$ of the original thinned sputum. Then it was heat inactivated at $80^{\circ} \mathrm{C}$ for 20 minutes in a dry heat block. After cooling to room temperature, an equal volume of chloroform was added and mixed by vortex and then stored in refrigerator ${ }^{16}$.

\section{DNA extraction}

The Mycobacterial DNA used for LAMP was extracted from concentrated sputum samples by freeze and boil method ${ }^{17}$. Briefly, $50 \mu \mathrm{l}$ of sample was taken into an eppendorf tube. Then it was immersed into liquid nitrogen $\left(-196^{\circ} \mathrm{C}\right)$ for one minute and it was heated to $100^{\circ} \mathrm{C}$ in the dry heat block for one minute. The alternate cooling and heating was repeated for five times to effect DNA extraction and the extracted DNA was stored in the refrigerator for LAMP and PCR. The concentration and quality of genomic DNA in PCR reaction were determined by spectrophotometric analysis at 260 and $280 \mathrm{~nm}^{18}$.

\section{Primer for LAMP}

For rapid diagnosis of tuberculosis, mycobacterial DNA extracted from the concentrated sputum sample was used for LAMP all together six primers were used during the study. These were: outer primers (F3 and B3), a forward inner primer (FIP), a backward inner primer (BIP), and loop primers (loop F and loop B). They recognize eight distinct regions of the 16SrRNA (Eiken Chemical)

FIP:

CACCCACGTGTTACTCATGCAAGTCGAACGGAAAGGTCT

BIP: TCGGGATAAGCCTGGACCACAAGACATGCATCCCGT

FL: GTTCGCCACTCGAGTATCTCCG

BL: GAAACTGGGTCTAATACCGG

F3: CTGGCTCAGGACGAACG

\section{B3: GCTCATCCCACACCGC}

\section{LAMP assay:}

LAMP reaction was performed in a total $25 \mu \mathrm{l}$ reaction mixture containing $2.5 \mu \mathrm{l} 10 \mathrm{x}$ LAMP buffer $(200 \mathrm{mM}$ TrisHCL [pH8.8], $10 \mathrm{mM} \mathrm{KCl,} 10 \mathrm{mM}$ (NH4)2SO4, 1\% Triton $\mathrm{X}-100,14 \mathrm{mM}$ dNTPs (Deoxynucleoside triphosphates), $0.8 \mathrm{M}$ betaine, $300 \mathrm{mM} \mathrm{MgSO}_{4}$, primer mix $(30 \mathrm{pmol}$ each of inner primers FIP and BIP, 5 pmol each of outer primer FL and BL, 20 pmol each of loop primers F3 and B3), 8U Bst DNA polymerase (New England Blolabs) with specified amounts of bacterial DNA, and Loopamp Fluorescent Detection Reagent (Eiken Chemical Co Ltd., Tokyo, Japan). Loopamp Fluorescent Detection Reagent enables the direct detection of DNA amplification in reaction tubes by naked eye as green fluorescence under ultraviolet light.

\section{Statistical analysis}

We assumed statistical significance at $p<0.05$. The sensitivity, specificity, positive predictive value and negative predictive values were calculated with a 95\% confidence interval (95\% CI) using the standard formulas considering culture as the gold standard.

\section{RESULTS}

In order to evaluate the different diagnostic techniques for rapid diagnosis of tuberculosis, a comparative study of LAMP and microscopy to detect M. tuberculosis in sputum sample, against culture as standard method was performed. The sputum samples 
from the patients belonging to two different study groups were collected in this study. Out of 106 samples, 53 were from smear positive TB patients and 53 were from smear negative patients respectively.

\section{Distribution of total study cases $(\mathrm{N}=106)$ by age and} sex

Among the studied 106 cases, 72.64\% (n=77/106) were males and $27.36 \%(n=29 / 106)$ were females in the age group 14 to 81 years. This study showed that the highest number was seen in the age group of 21 - 40 years (36.79\%) among males and in the age group $41-60$ (9.43\%) among females.

Table 1: Distribution of total study cases by age and sex

\begin{tabular}{ccccccc}
\hline Age & \multicolumn{2}{c}{ Males } & \multicolumn{2}{c}{ Females } & \multicolumn{2}{c}{ Total } \\
\hline Group (years) & No & $\%$ & No & $\%$ & No & $\%$ \\
$<20$ & 8 & 7.54 & 8 & 7.54 & 16 & 15.09 \\
$21-40$ & 39 & 36.79 & 9 & 8.49 & 48 & 45.28 \\
$41-60$ & 21 & 19.81 & 10 & 9.43 & 31 & 29.24 \\
$>60$ & 9 & 8.49 & 2 & 1.89 & 11 & 10.37 \\
Total & 77 & 72.64 & 29 & 27.36 & 106 & 100 \\
\hline
\end{tabular}

Age and sex distribution of culture, LAMP and PCR positive cases

Out of 106 sputum samples, 53.77\% (n=57/106) were culture positive, $52.83 \%(n=56 / 106)$ were LAMP positive. Among total culture positive isolates, $73.68 \%(n=42 / 57)$ were males and $26.31 \%(n=15 / 57)$ were females. The highest number of culture positive cases were belonged to the age group 21-40. Out of 56 LAMP positive isolates, $71.42 \%(n=40 / 56)$ were males and $28.57 \%(n=16 / 56)$ were females.

\section{Socio-demographic and disease characteristic of interviewed persons}

106 patients were interviewed personally through structured questionnaire. Among 106 interviewed patients, $47.16 \% \quad(n=50 / 106$ were smokers; $52.83 \%$ $(n=56 / 106)$ were non-smokers; $42.45 \%(n=45 / 106)$ had habit of taking alcohol and 54.71\% $(n=58 / 106)$ were non alcoholic; $27.35 \%(n=29 / 106)$ showed presence of TB in their family where $72.64 \%(n=77 / 106)$ showed absence of TB in their family members.

Out of total interviewed patients, $70.75 \%(n=75 / 106)$ were immunized by BCG vaccination, where as $29.25 \%$ $(n=31 / 106)$ were non-vaccinated. While asking the patients about the symptoms of TB, 74.52\% ( $n=79 / 106)$ had the symptoms of fever, $68.86 \%(n=73 / 106)$ had chest pain, 86.79\% (n=92/106) had cough, 71.69\% (n=76/106) had weight loss, and $34.90 \%(n=37 / 106)$ had hemoptysis respectively.

Table 2: Socio-demographic and disease characteristic of interviewed persons $(n=106)$

\begin{tabular}{lcr}
\hline $\begin{array}{c}\text { Socio-demographic } \\
\text { characteristics }\end{array}$ & $\begin{array}{c}\text { No. of } \\
\text { patients }\end{array}$ & Percentage \\
\hline Smoking & & \\
Smoker & 50 & $47.16 \%$ \\
Non-Smoker & 56 & $52.83 \%$
\end{tabular}

\begin{tabular}{|c|c|c|}
\hline Alcohol & & \\
\hline Habit of drinking al- & 45 & $42.45 \%$ \\
\hline Not & 58 & $54.71 \%$ \\
\hline
\end{tabular}

Family history of TB

$\begin{array}{ccc}\text { Present } & 29 & 27.35 \% \\ \text { Absent } & 77 & 72.64 \%\end{array}$

BCG

$\begin{array}{lll}\text { Vaccinated } & 75 & 70.75 \% \\ \text { Non-Vaccinated } & 31 & 29.25 \%\end{array}$

\section{Symptoms}

$\begin{array}{lll}\text { Fever } & 79 & 74.52 \% \\ \text { Chest pain } & 73 & 68.86 \% \\ \text { Cough } & 92 & 86.79 \% \\ \text { Hemoptysis } & 37 & 34.90 \% \\ \text { Weight loss } & 76 & 71.69 \%\end{array}$

X-Ray

$\begin{array}{lll}\text { Positive } & 55 & 51.89 \% \\ \text { Negative } & 51 & 48.11 \%\end{array}$

Evaluation of MTB-LAMP for the detection of $M$. tuberculosis in clinical specimens

Application of MTB-LAMP to clinical specimens was evaluated by comparing LAMP results with acid-fast smear tests and standard bacterial cultures as gold standard test of 106 patients in Nepal. Results are summarized in Table 3 .

In the LAMP, all the positive extraction controls and positive LAMP controls gave expected green color changed throughout the experiment, thus, validating the 
extraction procedure and LAMP reaction condition. Also all the negative extraction controls and the negative LAMP controls gave no bands and orange color respectively, hence, excluding any possibility of contamination.

In order to minimize the risk of contamination with exogenous $M$. tuberculosis DNA, these procedures were performed using disposable tubes and pipettes. In addition, a sample blank was taken through all the preparation steps to detect possible contaminations. Furthermore, DNA extraction and LAMP assay were performed in different laboratories.

Table 3: Comparative results of stain, PCR and LAMP with culture

\begin{tabular}{lcccccc}
\hline & \multicolumn{3}{c}{ Stain } & \multicolumn{3}{c}{ LAMP } \\
\cline { 2 - 7 } & +ve & -ve & Total & +ve & -ve & Total \\
\hline Culture +ve & 53 & 4 & 57 & 55 & 2 & 57 \\
Culture -ve & 0 & 49 & 49 & 1 & 48 & 49 \\
Total & 53 & 53 & 106 & 56 & 50 & 106 \\
Sensitivity & $92.98 \%$ & & & $96.49 \%$ & & \\
Specificity & $100 \%$ & & & $97.95 \%$ & \\
PV + & $100 \%$ & & & $98.21 \%$ & \\
PV - & $92.45 \%$ & & $96 \%$ & \\
\hline
\end{tabular}

PV+: predictive value of positive test, and PV-: predictive value of negative test

Fig 1. Visual inspection of LAMP products. The $M$. tuberculosis primers were used for this assay. Tubes: PC, positive control; NC, negative control (no DNA (Nucleic acid free water only)); 195B and 288B, positive samples; 365B and 486B, negative samples.

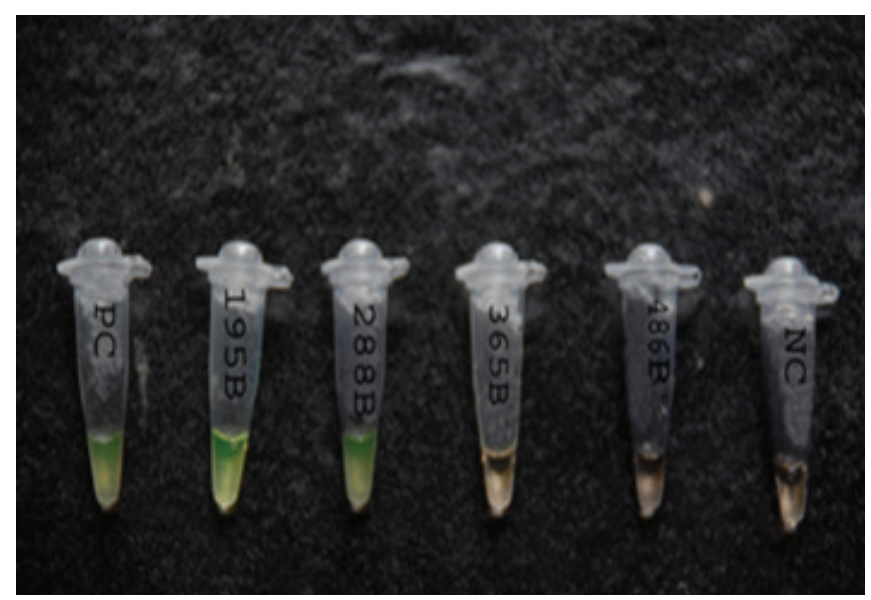

Among 53 smear positive cases; all were positive while culturing on Ogawa medium. However, among 53 smear negative cases, four were positive by culture. In this study, none of the fluorochrome staining positive samples showed negative results in culture, thus showing 100\% specificity. This means that as long as the stain gives positive result, it is not necessary to do further test for the purpose of diagnosis. However, due to the increase of drug resistant bacilli, culture is recommended to test for the sensitivity of antituberculosis drugs.

While in smear negative cases, the sensitivity of microscopy in reference to culture was found to $92.98 \%$. This indicates the sensitivity of microscopy was not good as its specificity. However, considering the availability and technical easiness, microscopy method could be still used as the method of choice in the first line diagnosis of tuberculosis.

Among 53 PTB patients with a positive acid fast stain on sputum smear and culture positive too, LAMP was positive for 51 samples while it was negative for the remaining two samples. While out of 53 smear negative samples from the patients of group B, LAMP was positive for five samples and it was negative for the rest of the samples. The finding reveals sensitivity of LAMP over microscopy. When the result of culture on Ogawa medium obtained, only four samples were found to be positive. These four culture positive samples were also positive by LAMP confirming the LAMP result with culture. These findings emphasized the sensitivity of the LAMP over culture. It is remarkable that LAMP positive sample was found to be negative by culture. 48 microscopy negative samples were found to be negative by both LAMP and culture. This finding highlighted the specificity of LAMP because all the samples that were negative for tubercle bacilli microscopically and culturally were also negative by LAMP. Thus, LAMP combines the rapidity of microscopy with specificity of culture.

While comparing the LAMP with culture as gold standard, the sensitivity specificity, predictive value of positive test, predictive value of negative test, percentage of false negative and percentage of false positive were $96.49 \%$, $97.95 \%, 98.21 \%, 96 \%, 3.50 \%$ and $2.04 \%$ respectively. The findings of the present study was in agreement with the previous findings of Boehme et $a l^{19}$ who reported the sensitivity of LAMP as $97.7 \%$ in comparison to culture as gold standard.

\section{Discussion}

Tuberculosis (TB) is a communicable disease caused by the bacterium Mycobacterium tuberculosis (MTB) and is a persistent problem in the developing countries. Early 
and effective detection of Mycobacterium tuberculosis, particularly in smear-negative tuberculosis, is a priority for global TB control. Detection of M. tuberculosis using conventional microscopy, culture and biochemical-based assays is time-consuming and laborious. Therefore, a simple and sensitive method for rapid detection that can be performed in any standard laboratory has been anxiously awaited to facilitate the initiation of clinical TB treatment. LAMP can be used for this purpose, providing results within $1 \mathrm{~h}$ with high sensitivity.

The objective of this study was to identify M. tuberculosis directly from sputum by LAMP and to compare its efficacy over routinely used methods. Sputum samples were collected from the patients visiting at the National TB Reference Lab German-Nepal Tuberculosis Project (GENETUP), Kalimati. Here in group A, a total of 53 sputum samples were collected from the new cases of pulmonary tuberculosis patients and in group $\mathrm{B}$, another total of 53 sputum specimens obtained from patients who were suspected of having TB were subjected to culture confirmation, smear microscopy and the LAMP method. The clinical sensitivity, specificity, positive predictive value (PPV) and negative predictive value (NPV) were calculated and compared with the culture results.

In this study, males $(72.64 \%)$ were found to be more infected than females $(27.36 \%)$ and the result was found to be significant statistically. This findings was in agreement with the findings of the Ponticeellio et $a 2^{20}$, who reported $82.2 \%$ males and $17.8 \%$ of females among 90 active pulmonary TB cases. This does not however reflect an increase in the occurrence of disease in males, since in present study the attendance of females is lower than males. TB was not diagnosed in the pulmonary TB suspects below 14 years. On the other hand females make less report than males in health care facilities, because of social pressure or stigma and males have more exposure to external environment than females for their job and other activities. In the present study maximum number of culture positive was observed in the economically most productive age group of $21-40$ years.

Among 53 pulmonary TB patients with a positive acid-fast stain on sputum smear, $94.33 \%(50 / 53)$ were positive by chest X-ray while the remaining were negative. The $\mathrm{X}$-ray positive results are due to cavities usually develops because of immune response to the tubercle bacilli leading to destruction of lung tissue. The X-ray negative result may be due to the people who don't have fully functioning immune system like in HIV cases, where there is less tissue destruction and hence lung cavitations. Another reason might be due to the patients who were suffered from bronchial or tracheal tuberculosis in whom infectious agent might not be spread to the lungs.

Patients with negative acid fast staining on sputum samples were recruited into group B where 9.43\% (5/53) were positive in chest X-ray and the rest were negative. Abnormalities on chest X-ray may be suggestive of, but are never diagnostic of TB because a number of other bacterial conditions (such as pneumonia or abscess) or non-bacterial processes (fungal diseases, carcinoma, sarcoidosis or pneumoconiosis) can produce similar images. The vast majority of patients (over 90\%) with cavitary pulmonary TB are sputum smear positive (WHO, 1997 ${ }^{21}$.

Among 53 smear positive cases; all were positive while culturing on Ogawa medium. However, among 53 smear negative cases, four were positive by culture. In this study, none of the fluorochrome staining positive samples showed negative results in culture, thus showing 100\% specificity. This means that as long as the stain gives positive result, it is not necessary to do further test for the purpose of diagnosis. However, due to the increase of drug resistant bacilli, culture is recommended to test for the sensitivity of antituberculosis drugs ${ }^{22}$.

While in smear negative cases, the sensitivity of microscopy in reference to culture was found to $92.98 \%$. This indicates the sensitivity of microscopy was not good as its specificity. However, considering the availability and technical easiness, microscopy method could be still used as the method of choice in the first line diagnosis of tuberculosis ${ }^{23}$.

Among 53 PTB patients with a positive acid fast stain on sputum smear and culture positive too, LAMP was positive for 51 samples while it was negative for the remaining two samples. While out of 53 smear negative samples from the patients of group B, LAMP was positive for five samples and it was negative for the rest of the samples. The finding reveals sensitivity of LAMP over microscopy. When the result of culture on Ogawa medium obtained, only four samples were found to be positive. These four culture positive samples were also positive by LAMP confirming the LAMP result with culture. These findings emphasized the sensitivity of the LAMP over culture. It is remarkable that LAMP positive sample was found to be negative by culture. Forty eight microscopy 
negative samples were found to be negative by both LAMP and culture. This finding highlighted the specificity of LAMP because all the samples that were negative for tubercle bacilli microscopically and culturally were also negative by LAMP. Thus, LAMP combines the rapidity of microscopy with specificity of culture.

While comparing the LAMP with culture as gold standard, the sensitivity specificity, predictive value of positive test, predictive value of negative test, percentage of false negative and percentage of false positive were $96.49 \%$, $98.21 \%, 98.21 \%, 96 \%, 3.50 \%$ and $2.04 \%$ respectively. This demonstrates a high sensitivity, specificity and predictive value of positive test and predictive value of negative test of LAMP as compared with that of culture. Percentage of false negative and percentage of false positive are also very low indicating the higher accuracy of the test.

LAMP-based assays targeting the gyrB (Iwamoto et al. 2003), 16S rRNA (Pandey et al. 2008) ${ }^{23}$, IS6110 (Aryan et al. 2010) ${ }^{24}$ and rimM (Zhu et al. 2009) ${ }^{25}$ sequences have been developed for the detection of $M$. tuberculosis. A multicenter study of Mycobacterium tuberculosis detection showed the feasibility of using the LAMP method in developing countries by Boehme et a $1^{19}$. They had shown the sensitivity of MTB-LAMP 97.7\% (173/177) in smear positive and culture positive sputum samples and $48.8 \%$ (21/43) in smear negative and culture positive sputum samples.

The findings of the present study was in agreement with the findings of Pandey et al on Nepalese patients demonstrated the feasibility of LAMP for the detection of M. tuberculosis. They had shown the sensitivity of LAMP $100 \%(96 / 96)$ in culture positive sputum sample, and the specificity was $94.2 \%(98 / 104)$ in culture negative sputum samples. Similarly, Cathrina et a ${ }^{26}$ reported that the sensitivity of LAMP in smear and culture positive sputum specimen was $97.7 \%$ and sensitivity in smear negative and culture positive specimen was $48.8 \%$. The specificity in culture negative sample was $99.0 \%$.

\section{CONCLUSIONS}

In conclusion, this study showed that LAMP test is not necessary for the diagnosis of smear positive cases. However LAMP could be possible tool for confirmatory diagnosis of the smear negative cases, which show clinical symptoms of TB. Among the different diagnostic tools,
LAMP assay is more advantageous than other techniques performed in this study due to its easy operation without sophisticated equipment, it will be simple enough to use in small-scale hospitals, primary care facilities, and clinical laboratories in developing countries if the remaining issues such as sample preparation, nucleic acid extraction, and cross-contamination controls are addressed.

\section{REFERENCES}

1. World Health Organization. Global tuberculosis report 2015. Available from http://www.who.int/tb/ publications/global_report/en/.

2. Elliott AM, Halwiindi B, Hayes RJ, Luo N, Tembo G et $a l$. The impact of human immunodeficiency virus on presentation and diagnosis of tuberculosis in a cohort study in Zambia. J Trop Med Hyg 1993; 96: 1-11.

3. Klein NC, Duncanson FP, Lenox TH 3rd, Pitta A, Cohen $\mathrm{SC}$ et al. Use of mycobacterial smears in the diagnosis of pulmonary tuberculosis in AIDS/ ARC patients. Chest. 1989; 95: 1190-1192.

4. Hofmann-Thiel S, Turaev L, Hoffmann H. Evaluation of the hyplex TBC PCR test for detection of Mycobacterium tuberculosis complex in clinical samples. BMC Microbiol. 2010; 10: 95.

5. WHO (2013a). Global Tuberculosis Report 2013. Geneva: World Health Organization.

6. Abe C, Hirano K, Wada M, Kazumi Y, Takahashi M et al. Detection of Mycobacterium tuberculosis in clinical specimens by polymerase chain reaction and GenProbe Amplified Mycobacterium Tuberculosis Direct Test. J Clin Microbiol. 1993; 31: 3270-3274.

7. Helb D, Jones M, Story E, Boehme C, Wallace E et al. Rapid detection of Mycobacterium tuberculosis and rifampin resistance by use of on-demand, nearpatient technology. J Clin Microbiol. 2010; 48: 229237.

8. Guo Y, Zhou Y, Wang C, Zhu L, Wang S et al. Rapid, accurate determination of multidrug resistance in M. tuberculosis isolates and sputum using a biochip system. Int J Tuberc Lung Dis. 2009; 13: 914-920.

9. Huggett JF, McHugh TD, Zumla A. Tuberculosis: Amplification-based clinical diagnostic techniques. Int J Biochem Cell Biol. 2003; 35: 1407-1412. 
10. Sarmiento OL, Weigle KA, Alexander J, Weber DJ, Miller WC. Assessment by meta-analysis of PCR for diagnosis of smear-negative pulmonary tuberculosis. J Clin Microbiol. 2003; 41: 3233-3240.

11. Suffys P, Palomino JC, Cardaso Lea o S, Espitia C, Cataldi A et al. Evaluation of the polymerase chain reaction for the detection of Mycobacterium tuberculosis. Int J Tuberc Lung Dis. 2000; 4: 179-183.

12. Notomi T, Okayama H, Masubuchi H, Yonekawa $\mathrm{T}$, Watanabe $\mathrm{K}$ et al. Loop-mediated isothermal amplification of DNA. Nucleic Acids Res. 2000; 28: 63-63.

13. Iwamoto, T., Sonobe, T. \& Hayashi, K. Loop-mediated isothermal amplification for direct detection of Mycobacterium tuberculosis complex, M. avium, and M. intracellulare in sputum samples. J Clin Microbiol. 2003; 41, 2616-2622.

14. WHO (1998a). Laboratory services in tuberculosis control. Part II: Microscopy. World Health Organization, Geneva, Switzerland.

15. WHO (1998b). Laboratory services in tuberculosis control. Part III: culture. World Health Organization, Geneva, Switzerland.

16. Sapkota BR, Ranjit C, Macdonald M. Rapid differentiation of Mycobacterium tuberculosis and Mycobacteium leprae from sputum by polymerase chain reaction. Nepal Med Coll J. 2007; 9(1): 12-16.

17. Woods SA, Cole ST. A rapid method for the detection of potentially viable Mycobacterium leprae in human biopsies: a novel application of PCR. FEMS Microbiology Letters. 1989; 65: 305-310.

18. Sambrook J, Fritsch EF and Maniatis T (1989). Molecular cloning: a laboratory manual, 2nd edn. Cold spring Harbor Laboratory Press, USA.

19. Boehme CC et al. Operational feasibility of using loopmediated isothermal amplification for diagnosis of pulmonary tuberculosisin microscopy centers of developing countries. J Clin Microbiol. 2007; 45:
1936-1940.

20. Ponticeellio A, Perna F, Sturbenboom MCJM, Etiello IM, Bocchino M and Sanduzzi A. Demographic risk factors and lymphocyte populations in patients with tuberculosis and their healthy contacts. Int J Tuberc Lung Dis. 2001; 5: 1148-1155.

21. WHO (1997) TB: A clinical manual for South East Asia. World Health Organization, Geneva, Switzerland, pp 65.

22. Sohn KY, Shrestha S, Khagi A, Malla SS, Pokhrel BM, Khanal MP, Rijal B, Bajracharya P. Polymerase chain reaction detection of Mycobacterium tuberculosis from sputum. J Nep Med Assoc. 2003; 42: 65-70.

23. Pandey BD, Poudel A, Yoda T, Tamaru A, Oda N, Fukushima Y, Lekhak B, Risal B, Acharya B et al. Development of an in-house loop-mediated isothermal amplification (LAMP) assay for detection of Mycobacterium tuberculosis and evaluation in sputum samples of Nepalese patients.J Med Microbiol. 2008; 57: 439-443.

24. Aryan E, Makvandi M, Farajzadeh A, Huygen K, Bifani P, Mousavi SL, Fateh A, Jelodar A, Gouya MM, Romano M. A novel and more sensitive loop-mediated isothermal amplification assay targeting IS6110 for detection of Mycobacterium tuberculosis complex. Microbiol Res. 2010; 165: 211-220.

25. Zhu RY, Zhang KX, Zhao MQ, Liu YH, Xu YY, Ju CM, Li B, Chen JD. Use of visual loop-mediated isotheral amplification of rimM sequence for rapid detection of Mycobacterium tuberculosis and Mycobacterium bovis. J Microbiol Methods. 2009; 78: 339-343.

26. Catharina CB, Pamela N, German H, Rubhana R, Zeaur R, Martina G, Erica S, Michael H, Tsugunori N, Testu $\mathrm{H}$ and Mark DP. Operational feasibility of using loopmediated isothermal amplification for diagnosis of pulmonary tuberculosis in microscopy centers of developing countries. J Clinc Microbiol. 2007; 45(6): 1936-1940. 